\title{
Molecular Dynamics Simulation of a Polymer Melt with a Nanoscopic Particle
}

\section{Francis W. Starr,* Thomas B. Schrøder, ${ }^{\dagger}$ and Sharon C. Glotzer ${ }^{\ddagger}$}

Polymers Division and Center for Theoretical and Computational Materials Science, National Institute of Standards and Technology, Gaithersburg, Maryland 20899

Received April 10, 2001; Revised Manuscript Received December 3, 2001

\begin{abstract}
We perform molecular dynamics simulations of a bead-spring polymer melt surrounding a nanoscopic particle. We explore the effect of the polymer/nanoparticle interactions, surface-to-volume ratio, and boundary conditions on both the structure and dynamics of the polymer melt. We find that the chains near the nanoparticle surface are elongated and flattened and that this effect is independent of the interaction for the range of interactions we study. We show that the glass transition temperature $\mathrm{T}_{\mathrm{g}}$ of the melt can be shifted to either higher or lower temperatures by tuning the interactions between polymer and nanopartide. A gradual change of the polymer dynamics approaching the nanopartide surface causes the change in the glass transition. The magnitude of the shift is exaggerated by increasing fraction of surface monomers in the system. These behaviors support a "many-layer"-based interpretation of the dynamics. Our findings appear applicable to systems in which surface interactions dominate, including both traditional and nanofilled polymer melts, as well as systems with markedly different geometries, such as ultrathin polymer films. In particular, we show how our results might be compared with those obtained from experimental studies of "bound" polymer.
\end{abstract}

\section{Introduction}

Traditional fillers, such as carbon black, talc, and silica, have long been used to improve the properties of polymer materials ${ }^{1,2}$ and have been widely used for industrial purposes. The innovation of new materials remains an important goal, particularly in rapidly growing areas such as micro- and nanoelectronic devices. ${ }^{1,3}$ The need for new materials has driven an increased interest in understanding, at a molecular level, the changes in structural and dynamic properties caused by fillers and more recently by "nanoparticles". Nanoparticles, such as gold particles, ${ }^{4}$ silica beads or silicate nanoparticles, ${ }^{1,5}$ fullerenes and nanotubes, ${ }^{6}$ silsesquioxanes, ${ }^{3,7}$ and others ${ }^{8}$ have the potential to be far more homogeneously distributed than traditional fillers and in some cases allow for specifically designed shape and functionality, influencing the material properties at molecular scales. ${ }^{3}$

Molecular simulations provide an excellent opportunity to directly study the influence of nanoparticles on structure and dynamics of polymers, since detailed information on the properties near a nanoparticle surface is difficult to obtain experimentally. In this paper, we study a model nanoparticle embedded in a dense melt of unentangled polymers via molecular dynamics simulation. We first probethe changes in melt structure due to the nanoparticle, which we find to be largely insensitive to the interactions between the nanoparticle and the polymers. The majority of our results focus on the changes in dynamics caused by the nanoparticle. We show the extent to which interactions between polymer chains and the nanoparticle, as well as the boundary conditions, change the glass transition

\footnotetext{
† Present address: IMFUFA, Roskilde University, DK-4000 Roskilde, Denmark.

₹ Present address: Departments of Chemical Engineering and Materials Science and Engineering, University of Michigan, Ann Arbor, MI 48109.
}

temperature $T_{g}$ and the average and local dynamic properties of the melt. These results help rationalize experiments on filled polymers that report both increases and decreases of $T_{g}$, depending on the details of the system studied. ${ }^{1,9-11} \mathrm{~A}$ brief report of some of these results recently appeared in ref 12 . An additional simulation study of a nanofilled polymers also recently appeared in ref 13.

Our results show a strong similarity to those previously found for ultrathin films and polymers near simple surfaces. As a result, it may be possible to interpret the changes induced by nanofillers in the same framework used to interpret the behavior of ultrathin polymer films and simple surfaces. In particular, ultrathin films have many important technological applications (e.g., paints, lubricants, adhesives, and electronic packaging), and the causes of changes in the structure and dynamics are a topic of continuing discussion; ${ }^{14-20,22-28}$ our nanofiller results may contribute to this discussion.

We organize the paper as follows: in section 2 we describe the details of the simulation model and method. We focus on the structural changes induced by the nanoparticle in section 3 and on dynamic properties in section 4 . In section 5 we discuss how our results relate to ultrathin film systems, where surface interactions also play an important role. We then expand our results by considering the effect of a free surface in section 6 and compare with experimentally studied "bound" polymers. Finally, we discuss the implications of our results and the role of surface interactions vs confinement in section 7 .

\section{Simulations}

Our findings are based on molecular dynamics simulations of a single nanoscopic particle surrounded by a dense polymer melt as well as simulations of a pure melt for comparison purposes. We use a well-studied beadspring model $29-31$ which models polymers as chains of monomers. All monomer pairs interact via a Lennard- 
J ones (LJ ) potential $V_{L J}$, and bonded monomers along a chain are connected via a FENE anharmonic spring potential

$$
\mathrm{V}_{\mathrm{FENE}}=-\mathrm{k}\left(\mathrm{R}_{0}^{2} / 2\right) \ln \left(1-\left(\mathrm{r} / \mathrm{R}_{0}\right)^{2}\right)
$$

where $r$ is the distance between neighboring monomers, and $\mathrm{k}$ and $\mathrm{R}_{0}$ are adjustable parameters that we will discuss.

Several truncations of the LJ potential have been considered in previous works; Kremer and Grest truncated at the minimum of the $L J$ potential and shifted the potential so that it is zero and continuous at $2^{1 / 6} \sigma$ (where $\sigma$ is the LJ length parameter), resulting in a nearly athermal model. ${ }^{32}$ Bennemann et al. ${ }^{33}$ truncated and shifted the potential at $2 \cdot 2^{1 / 6} \sigma$, resulting in a system displaying a temperature-driven glass transition, as we also desire. However, the truncation used by Bennemann et al. has a discontinuity in the force at the cutoff distance $r_{c}$. An expression for the force without a discontinuity allows for direct potential energy minimization via a steepest descent in order to study the underlying potential energy surface, often used to characterize glass-forming systems; ${ }^{34,35}$ such a study of this model will be presented elsewhere. To avoid a discontinuity in the force, we use the so-called "shiftedforce" (sf) potential ${ }^{36}$ for $r<r_{c}$

$$
V_{s f}(r)=V_{L J}(r)-V_{L J}\left(r_{c}\right)-\left.\left(r-r_{c}\right) \frac{d V_{L J}(r)}{d r}\right|_{r=r_{c}}
$$

We choose $r_{c}=2.5 \sigma$. For $r>r_{c}, V_{s f}(r)=0$. We find that this potential results in a melt with no significant qualitative differences from those of ref 33. However, we point out that ref 33 studied chains of 10 monomers, while we focus on chains of 20 monomers.

We use reduced units in which $\mathrm{m}=\sigma=\epsilon=1$ ( $\epsilon$ is the $L J$ energy parameter and $\mathrm{m}$ is the monomer mass), since we do not aim to study a specific polymer, and we present our results in "reduced units", where length is in units of $\sigma$, time in units of $\sigma \sqrt{\mathrm{m} / \epsilon}$, and temperature in units $\epsilon / \mathrm{K}_{B}$ ( $\mathrm{K}_{\mathrm{B}}$ is Boltzmann's constant). The parameters of theFENE potential are $k=30$ and $R_{0}=1.5$, as used in ref 33. This choice introduces incompatible length scales in the inter- and intrapolymer interactions; this incompatibility inhibits crystallization, allowing us to study the slow dynamics approaching the glass transition. Furthermore, this choice ensures relatively stiff bonds and avoids very high-frequency modes and chain crossing. The average bond length for these parameter choices is narrowly distributed around 0.96 .

For the pure melt, we simulate 100 chains of $M=20$ monomers each (a total of $\mathrm{N}=2000$ monomers), bel ow the entanglement length, but long enough that chains exhibit Gaussian statistics, in the sense that the endto-end distance $R_{e}^{2}=6 R_{g}{ }^{2}$, where the radius of gyration is defined by ${ }^{37}$

$$
\left.R_{g}^{2}=\frac{1}{M^{2}} \mid \sum_{i, j=0}^{M}\left(r_{i}-r_{j}\right)^{2}\right)
$$

where $\mathbf{r}_{\mathrm{i}}$ is the position of monomer $\mathrm{i}$ and $\langle\ldots\rangle$ denotes an average over chains and configurations. We study state points in the range $T=0.35-4.0$ at a constant density of $\rho=1.0$. Periodic boundary conditions are used in all directions. We summarize the thermodynamic and
Table 1. Summary of the Simulations of the Pure Melta

\begin{tabular}{ccccc}
\hline$T$ & $\mathrm{U}$ & $\tau$ & $\mathrm{t}_{\text {eq }}$ & $\mathrm{t}_{\mathrm{pr}}$ \\
\hline 0.350 & 14.65 & 678 & $3.3 \times 10^{4}$ & $5 \times 10^{4}$ \\
0.370 & 14.68 & 285 & $5 \times 10^{3}$ & $3 \times 10^{4}$ \\
0.401 & 14.73 & 132 & $2.6 \times 10^{3}$ & $4 \times 10^{3}$ \\
0.423 & 14.77 & 72.2 & $1.5 \times 10^{3}$ & $3 \times 10^{3}$ \\
0.447 & 14.81 & 45.9 & $2 \times 10^{3}$ & $1 \times 10^{3}$ \\
0.495 & 14.89 & 22.6 & $1.5 \times 10^{3}$ & $1 \times 10^{3}$ \\
0.583 & 15.04 & 10.2 & $1.5 \times 10^{3}$ & $3 \times 10^{2}$ \\
0.693 & 15.20 & 5.38 & $3 \times 10^{2}$ & $4 \times 10^{2}$ \\
0.795 & 15.35 & 3.84 & $1 \times 10^{3}$ & $4 \times 10^{2}$ \\
0.903 & 15.57 & 2.74 & $6 \times 10^{2}$ & $4 \times 10^{2}$ \\
1.005 & 15.65 & 2.19 & $1 \times 10^{3}$ & $2 \times 10^{2}$ \\
1.256 & 15.98 & 1.55 & $1 \times 10^{2}$ & $2 \times 10^{2}$ \\
1.510 & 16.30 & 1.15 & $4 \times 10^{2}$ & $1 \times 10^{2}$
\end{tabular}

a All simulations consist of 100 chains of 20 monomers at density 1.0. $U$ is the average potential energy per particle, and $\tau$ is the relaxation time of the intermediate scattering function of the monomers at the q-vector corresponding to the maximum in the structure factor. State points are equilibrated for a time teq, followed by production runs of duration $t_{p r}$; the number of time steps may be recovered by dividing by the incremental time step of 0.002 . We average dynamic properties over multiple possible starting times to reduce noise. Note that at $\mathrm{T}=0.35$ and 0.37 , we perform two independent runs to further improve statistics.

simulation details of each state point corresponding to the pure melt in Table 1.

We wish to model the nanoparticle with sufficient detail that it has general features typical of both traditional filler particles, ${ }^{1,2}$ such as a primary carbon black particle, and newer nanoparticles. ${ }^{3-7}$ Therefore, we design the nanoparticle such that (i) it has a size of order $10 \mathrm{~nm}$ and (ii) it is highly faceted and symmetric, such as gold nanoparticles; ${ }^{4}$ specifically, we choose an icosahedral shape for the nanoparticle.

To maintain the icosahedral shape, we assign ideal force sites at the vertices, at four equidistant sites al ong each edge of length $I=5 \cdot 2^{1 / 6}, 38$ and at six symmetric sites on the interior of each face of the icosahedron, forming a triangular lattice, as shown in Figure la. The size of the facets is chosen roughly equal to the end-toend distance $R_{e}$ of the polymers comprising the surrounding melt, which is $\mathrm{O}(10 \mathrm{~nm})$. We tether a LJ particle to each of these sites by a FENE spring to maintain a relatively rigid structure, which allows for thermalization and a small surface roughness of the nanoparticle; for the tether we use a stiffer spring ( $k_{p}$ $=45)$, larger mass $\left(m_{p}=2 m\right)$, and smaller bond length parameter $\left(R_{0}^{p}=1.0\right)$ than used for the polymers to reduce the fluctuation of nanoparticle sites. F or interactions between the nanoparticle force sites, we use the same form of LJ interaction as used for nonbonded monomers, given in eq 2 . We choose the nanoparticle site size $\sigma_{\mathrm{p}}=\sigma$, and the strength of the particle-particle interactions $\epsilon_{\mathrm{pp}}=2 \epsilon$. The nanoparticle site spacing of $2^{1 / 6}$, the preferred distance of the $L J$ potential, is small enough to prevent chains from penetrating the nanoparticle surface, except at very high density or temperature. To avoid any spurious effects that might be induced by a large cavity inside the nanoparticle, a replica of the particle with three sites on an edge and one with only sites at the vertexes are layered inside the outer icosahedron, reminiscent of a "Russian doll". This yields a total 356 force sites associated with the nanoparticle.

To determine which properties are a result of the steric constraints (excluded volume) imposed by the nanoparticle and which properties are affected by monomer-particle attraction, we consider a system with 

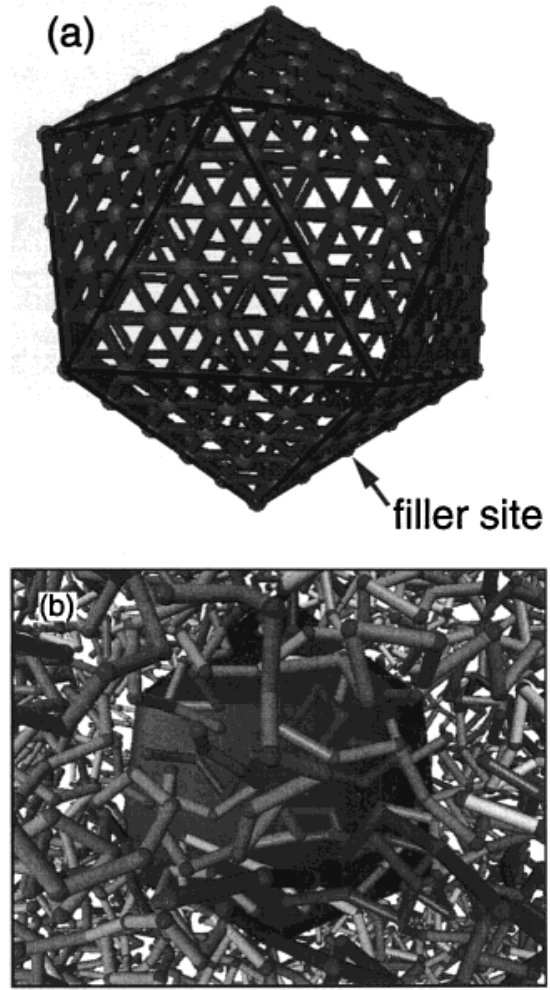

Figure 1. (a) Schematic showing the locations of the ideal force sites associated with the nanoparticle. The dark lines are to aid in distinguishing the faces of the icosahedron. (b) "Snapshot" of our simulation of the filled polymer melt. The bonds between nearest-neighbor monomers al ong a chain are drawn in various shades of gray for clarity.

a nanoparticle having only an excluded-volume interaction as well as a system with a nanoparticle having excluded-volume and attractive interactions. We choose the same parameters for the interaction potential for all nanoparticle force sites. ${ }^{39}$ We also use the LJ interaction described by eq 2 for monomer-nanoparticle interactions in the case of the attractive nanoparticle; for excluded-volume interactions only, we drop the $r^{6}$ attractive term in the $L J$ potential and leave all other parameters unchanged. For monomer-particle interactions, we use the Lorentz-Berthel ot ${ }^{36}$ mixing rules $\epsilon_{\mathrm{mp}}=\sqrt{\epsilon_{\mathrm{mm}} \epsilon_{\mathrm{pp}}}$. Our choice yields monomer-particle interactions that are stronger than monomer-monomer interactions, as expected for many nanoparticles and fillers. A snapshot of the simulation is shown in Figure $1 b$.

We simulate systems with 400,200 , and 100 chains of $\mathrm{M}=20$ monomers each (for totals of $\mathrm{N}=8000,4000$, and 2000 monomers) to address the effect of varying the surface-to-volume ratio of polymers. To compare the simulations of the filled system with the pure melt, we must be careful that the density of monomers far from the nanoparticle coincides with that of the pure system, since a slight change in density can cause a trivial, but significant, change in the structural and especially dynamic properties of the system. In this way, we probe only the changes caused by the interactions with the nanoparticle. A series of preliminary simulations was carried out to determine the box size so that the local density far from the nanoparticle deviates at most by $0.2 \%$ from the density of the pure melt; ref 33 studied the effect of density on $\mathrm{T}_{\mathrm{g}}$ (actually, the closely related $\mathrm{T}_{\mathrm{MCT}}$ ), and a density difference of $0.2 \%$ causes a change in $T_{g}$ less than those we report from the effect of
Table 2. Summary of the Simulations of the Polymer/ Nanoparticle System for Attractive Polymer-Particle Interactions ${ }^{a}$

\begin{tabular}{cclcrrr}
\hline $\mathrm{T}$ & $\mathrm{N}_{\mathrm{p}}$ & \multicolumn{1}{c}{$\mathrm{L}$} & $\mathrm{U}$ & \multicolumn{1}{c}{$\tau$} & \multicolumn{1}{c}{$\mathrm{t}_{\mathrm{eq}}$} & \multicolumn{1}{c}{$\mathrm{t}_{\mathrm{pr}}$} \\
\hline 0.35 & 400 & 20.4 & 13.73 & 2166 & $1.2 \times 10^{4}$ & $3.6 \times 10^{4}$ \\
0.37 & 400 & 20.4 & 13.76 & 928 & $9 \times 10^{3}$ & $1.8 \times 10^{4}$ \\
0.40 & 400 & 20.4 & 13.80 & 386 & $7.8 \times 10^{3}$ & $1.8 \times 10^{4}$ \\
& 200 & 16.51 & 12.97 & 951 & $1.8 \times 10^{4}$ & $6 \times 10^{4}$ \\
0.42 & 400 & 20.4 & 13.85 & 180 & $7.2 \times 10^{3}$ & $3 \times 10^{3}$ \\
0.45 & 400 & 20.4 & 13.90 & \multicolumn{1}{c}{87.5} & $6 \times 10^{3}$ & $1.5 \times 10^{3}$ \\
& 100 & 13.58 & 11.56 & 671 & $1.8 \times 10^{4}$ & $3.6 \times 10^{4}$ \\
0.50 & 400 & 20.4 & 13.99 & 40.5 & $6 \times 10^{3}$ & $1.5 \times 10^{3}$ \\
& 200 & 16.51 & 13.16 & 65.1 & $1.2 \times 10^{4}$ & $4.8 \times 10^{4}$ \\
& 100 & 13.58 & 11.65 & 135.5 & $1.2 \times 10^{4}$ & $1.8 \times 10^{4}$ \\
0.55 & 400 & 20.4 & 14.08 & 21.2 & $3 \times 10^{3}$ & $6 \times 10^{2}$ \\
0.60 & 400 & 20.4 & 14.61 & 13.1 & $3.6 \times 10^{3}$ & $6 \times 10^{2}$ \\
& 200 & 16.51 & 13.33 & 17.3 & $6 \times 10^{3}$ & $2.4 \times 10^{4}$ \\
& 100 & 13.58 & 11.84 & 23.2 & $6 \times 10^{3}$ & $1.8 \times 10^{4}$ \\
0.70 & 400 & 20.4 & 14.32 & 6.98 & $1.8 \times 10^{3}$ & $3 \times 10^{2}$ \\
& 200 & 16.51 & 13.50 & 8.46 & $6 \times 10^{3}$ & $2.4 \times 10^{4}$ \\
& 100 & 13.58 & 12.04 & 11.2 & $6 \times 10^{3}$ & $1.8 \times 10^{4}$ \\
0.80 & 400 & 20.4 & 14.48 & 4.60 & $1.2 \times 10^{3}$ & $1.8 \times 10^{2}$ \\
& 200 & 16.51 & 13.66 & 5.27 & $3 \times 10^{3}$ & $1.2 \times 10^{4}$ \\
& 100 & 13.58 & 12.19 & 5.71 & $3 \times 10^{3}$ & $1.2 \times 10^{4}$ \\
0.90 & 400 & 20.4 & 14.62 & 3.23 & $1.5 \times 10^{3}$ & $9 \times 10^{1}$ \\
& 200 & 16.51 & 13.81 & 4.05 & $3 \times 10^{3}$ & $1.2 \times 10^{4}$ \\
& 100 & 13.58 & 12.36 & 4.39 & $3 \times 10^{3}$ & $1.2 \times 10^{4}$ \\
1.00 & 400 & 20.4 & 14.77 & 2.59 & $1.2 \times 10^{3}$ & $9 \times 10^{1}$ \\
& 200 & 16.51 & 13.96 & 2.86 & $3 \times 10^{3}$ & $1.2 \times 10^{4}$ \\
& 100 & 13.58 & 12.51 & 3.03 & $2.4 \times 10^{3}$ & $1.2 \times 10^{4}$ \\
1.20 & 400 & 20.4 & 15.04 & 1.79 & $1.2 \times 10^{3}$ & $6 \times 10^{1}$ \\
1.50 & 400 & 20.4 & 15.43 & 1.31 & $1.2 \times 10^{3}$ & $6 \times 10^{1}$ \\
& 100 & 13.58 & 13.18 & 1.29 & $1.2 \times 10^{3}$ & $6 \times 10^{3}$
\end{tabular}

a At the two lowest $T$, two runs were performed to improve statistics. All chains have 20 monomers, and $N_{p}$ is the total number of polymers.

Table 3. Summary of the Simulations of the Polymer/ Nanoparticle System for Excluded-Volume Polymer-Particle Interactions ${ }^{\mathbf{a}}$

\begin{tabular}{cccccr}
\hline $\mathrm{T}$ & $\mathrm{L}$ & $\mathrm{U}$ & $\tau$ & $\mathrm{t}_{\mathrm{eq}}$ & \multicolumn{1}{c}{$\mathrm{t}_{\mathrm{pr}}$} \\
\hline 0.40 & 20.6 & 13.98 & 106 & $3 \times 10^{3}$ & $6 \times 10^{3}$ \\
0.45 & 20.58 & 14.07 & 40.3 & $1.8 \times 10^{3}$ & $1.2 \times 10^{3}$ \\
0.50 & 20.56 & 14.16 & 22.8 & $1.5 \times 10^{3}$ & $6 \times 10^{2}$ \\
0.60 & 20.54 & 14.32 & 9.83 & $1.2 \times 10^{3}$ & $3 \times 10^{2}$ \\
0.70 & 20.52 & 14.48 & 5.76 & $1.2 \times 10^{3}$ & $3 \times 10^{2}$ \\
0.80 & 20.51 & 14.63 & 4.09 & $1.2 \times 10^{3}$ & $3 \times 10^{2}$ \\
1.00 & 20.49 & 14.92 & 2.57 & $1.2 \times 10^{3}$ & $1.2 \times 10^{2}$
\end{tabular}

a All simulations are of 400 chains of 20 monomers each.

interactions. We summarize the box sizes and temperatures simulated in Tables 2 and 3.

We empl oy the rRESPA multiple time step al gorithm to improve simulation speed; 40 we use a basic time step of 0.002 and use a three-cycle velocity verlet version of rRESPA with the forces separated into bonded (fast) and nonbonded (slow) components. The temperature is adjusted using the Andersen method; ${ }^{36}$ for production runs, we wish to study the Newtonian dynamics present in the NVE ensemble. However, a small drift in the total energy (causing a slight increase in $\mathrm{T}$ ) due to the accumulation of round-off error is unavoidable in the long simulations we present; to avoid any increase in $\mathrm{T}$, we weakly couple the temperature to a Berendsen thermostat ${ }^{36}$ with a time constant 1000.

\section{Effect of Nanoparticle on Structure}

We first focus our attention on the effect the nanoparticle has on the melt structure. One of the simplest characterizations of the melt structure is the monomer pair distribution function $g(r)$. In Figure 2, we show $g(r)$ for the pure system and the filled systems with 8000 


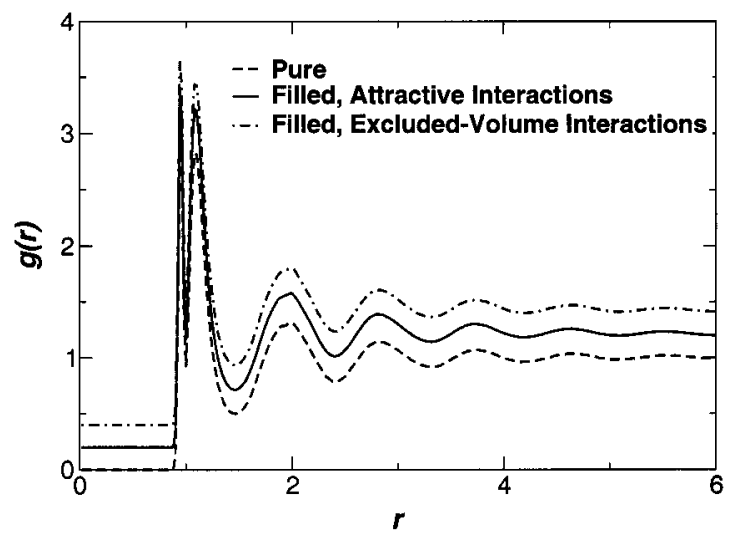

Figure 2. Pair distribution function $g(r)$ for each system studied at $T=0.4$. Successive curves are shifted by 0.2 on the vertical axis for clarity. When not shifted, the curves are nearly indistinguishable. Excluded-volume interactions correspond to the nonattractive case.

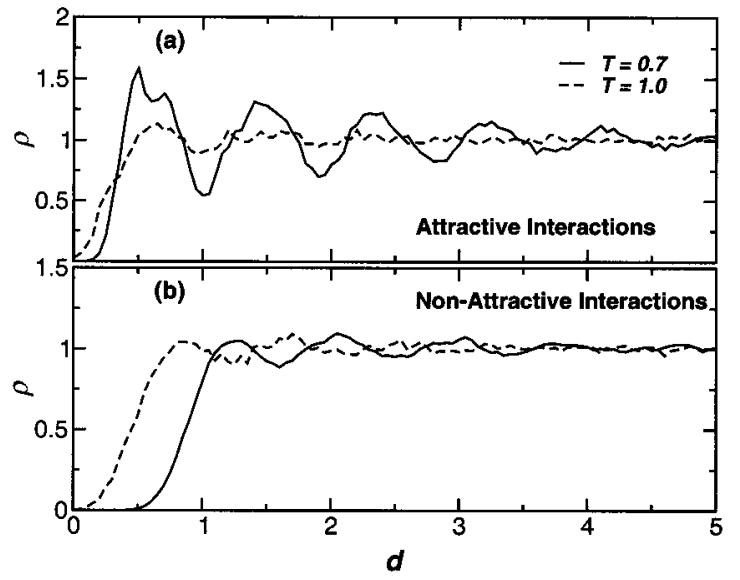

Figure 3. Monomer density profile $\rho(d)$ as a function of the distance from the nanoparticle surface for $\mathrm{T}=0.4$ and $\mathrm{T}=$ 1.0.

monomers at a low T, where we expect the structural differences are most pronounced since the thermal noise is reduced. There is no obvious difference in $g(r)$ between the filled and unfilled systems. Since the relative number of "surface" monomers is quite small in the 8000 monomer system, any differences in structure caused by surface effects are overwhelmed. However, even in the smaller filled systems (with a greater surface-tovolume ratio), we find that the differences in $g(r)$ are quite small.

3.1. Monomer Density Profile. To better understand the structural changes in the vicinity of the nanoparticle surface, we calculate the monomer density $\rho(d)$ as a function of the distance $d$ from the nanoparticle surface. Since the nanoparticle is icosahedral, it is nontrivial to calculate the distance of a monomer from the nearest surface. However, since the nanoparticle is nearly spherical, we may reasonably estimate $d=r-$ $r_{\text {surface, }}$ where $r$ is the radial position of a monomer relative to the particle center and $r_{\text {surface }}=(1 / 12)(42+$ $18 \sqrt{ } 5)^{1 / 2}$ is the radius of the inscribed sphere of the icosahedron. We show $\rho(d)$ for the filled system with (i) attractive and (ii) nonattractive (excluded vol ume only) interactions in Figure 3. In both cases, $\rho(d)$ has a welldefined layer structure. In the attractive case, we see a pronounced enhancement in the polymer density in the first layer, which we expect due to the relatively strong monomer-particle attraction; these density oscillations persist over a distance of roughly 4-5 monomers. N ot surprisingly, the density profile becomes better defined as $\mathrm{T}$ decreases. In the case of nonattractive interactions, there is an enhancement in the density in the first layer. However, notice that the location of the first layer is "pushed out" slightly, in comparison to the attractive nanoparticle case. The position of this peak increases with decreasing $\mathrm{T}$, since the monomers have less kinetic energy, limiting the distance of closest approach. The presence of a maximum in the absence of attraction is explained by the effective repulsion caused by the reduction in chain configurations near the wall, coupled with the dense melt pushing monomers back toward the surface, creating a "sandwiching" effect. ${ }^{21}$ The presence of a maximum and subsequent oscillations in $\rho(d)$ for both the attractive and nonattractive cases are consistent with the density profile of monomers observed near a smooth wall.18-21

3.2. Chain Conformation. The changes in the density profile must also be accompanied by some change in the local packing of the polymer chains, to which $g(r)$ is clearly insensitive. Other measures of the average structure, such as $\left\langle R_{\mathrm{g}}\right\rangle,\left\langle\mathrm{R}_{\mathrm{e}}\right\rangle$, and distribution of bond lengths and angles, show no significant deviations from the pure system. However, by focusing on the dependence of $R_{g}$ (or $R_{e}$ ) on the distance $d$ of the center-of-mass of a chain from the nanoparticle surface, we find a change in the overall polymer structure near the surface. We also resol ve the radial component $\mathrm{R}_{\mathrm{g}}^{\perp}$ of $R_{\mathrm{g}}$ relative to the nanoparticle center (approximately the component perpendicular to the particle surface). We obtain the radial component by substituting the segment vector $\mathbf{r}_{\mathrm{i}}-\mathbf{r}_{\mathrm{j}}$ in eq 3 with the dot product of the segment vector and the normalized bisector of each chain segment relative to the particle center, yielding

$$
R_{g}^{\perp 2}=\frac{1}{M^{2}}\left(\sum_{i, j=0}^{M}\left(\frac{\left(\mathbf{r}_{i}-\mathbf{r}_{j}\right) \cdot\left(\mathbf{r}_{i}+\mathbf{r}_{j}\right)}{\left|\mathbf{r}_{i}+\mathbf{r}_{j}\right|}\right)^{2}\right)
$$

Note that this equation assumes the filler center is located at the origin. In Figure 4, we show $\mathrm{R}_{\mathrm{g}}{ }^{2}$ and $\mathrm{R}_{\mathrm{g}}^{\perp 2}$ for both attractive and nonattractive monomer-particle interactions at one temperature. $\mathrm{R}_{\mathrm{g}}{ }^{2}$ increases by about $25 \%$ on approaching the particle surface; at the same time $\mathrm{R}_{\mathrm{g}}^{\perp 2}$ decreases by slightly more than a factor of 2 for both attractive and nonattractive systems. The combination of these results indicates that the polymers become slightly el ongated near the surface and flatten significantly, orienting with the particle surface. Note that not all monomers belonging to a given "surface polymer" are located in the first surface layer, as depicted in Figure 5 . We also point out that the chains retain a Gaussian conformation near the nanoparticle surface, in the sense that $R_{e}^{2}=6 R_{g}^{2}$; however, the probability distribution of the monomer coordinates is not symmetric in all directions.

The range of the flattening effect roughly spans a distance $R_{g}$ from the surface, and the results depend only weakly on $\mathrm{T}$. We also performed an additional simulation with double the attraction strength between the nanoparticle and polymers and did not find any significant further effect on the chain structure. The independence of the chain structure on the interaction 


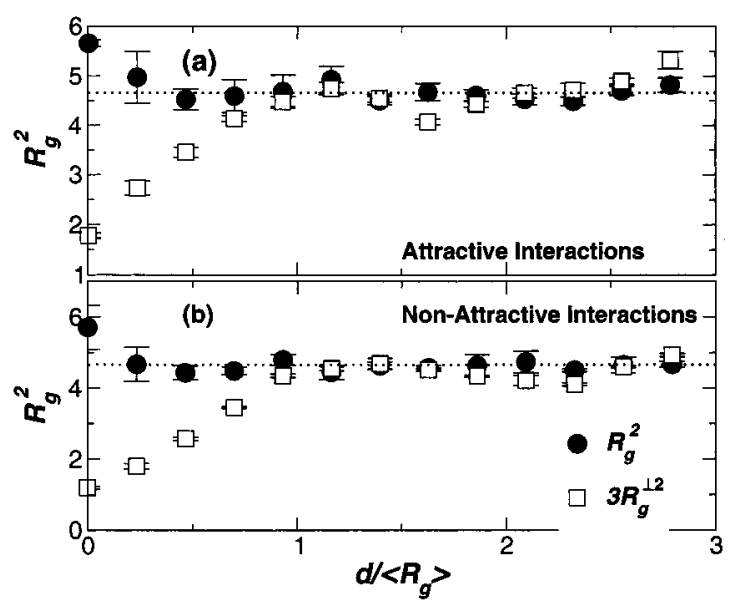

Figure 4. Radius of gyration $R_{g}$ of the polymer chains as a function of distance $d /\left\langle R_{g}\right\rangle$ of the center of mass of a chain from the nanoparticle surface for $T=0.4$. We also resolve the component perpendicular to the surface, which we label by $\mathrm{R}_{\mathrm{q}}^{\perp}$. We show results for (a) attractive and (b) nonattractive interactions. The dotted line shows $\left\langle R_{g}{ }^{2}\right\rangle$ for the pure system. The increase of $R_{g}$, coupled with the decrease of $R_{q}^{\perp}$, indicates that the chains become increasingly el ongated and "flattened" as the surface of the nanoparticle is approached. The effect appears largely independent of the temperature and numerical values of the potential parameters.

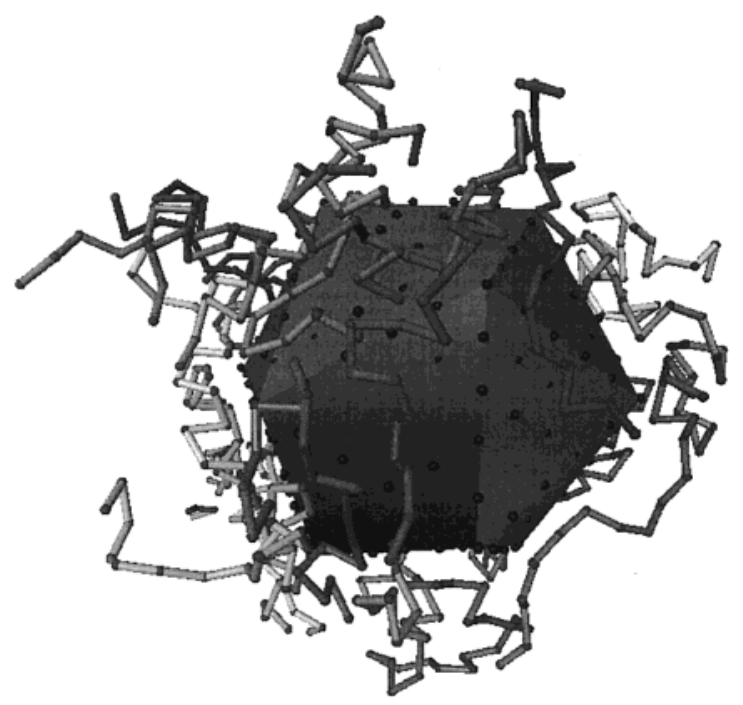

Figure 5. A few representative polymers that have monomers near the nanoparticle surface.

suggests that the altered shape of the polymers is primarily due to geometric constraints of packing the chains close $\left(\mathrm{d} \leqslant \mathrm{R}_{\mathrm{g}}\right)$ to the surface. In other words, the surface provides a preferential orientation for the polymers, and it is natural that this effect should persist for a distance $R_{g}$, roughly corresponding to the chain size. These results are also reminiscent of the properties of polymers near a wall and polymer thin films, despite the comparable size of chains and the nanoparticle, which we will discuss in detail in section 5 .

In the case that the interactions with the nanoparticle are significantly stronger and longer ranged, the observed effect on chain structure may be quite different. For an attractive interaction, increasing the strength and range would likely exaggerate the current effect; conversely, for a long-ranged repulsion, the chain might be el ongated perpendicular to the surface, similar to a polymer brush. We expect such alteration in struc-
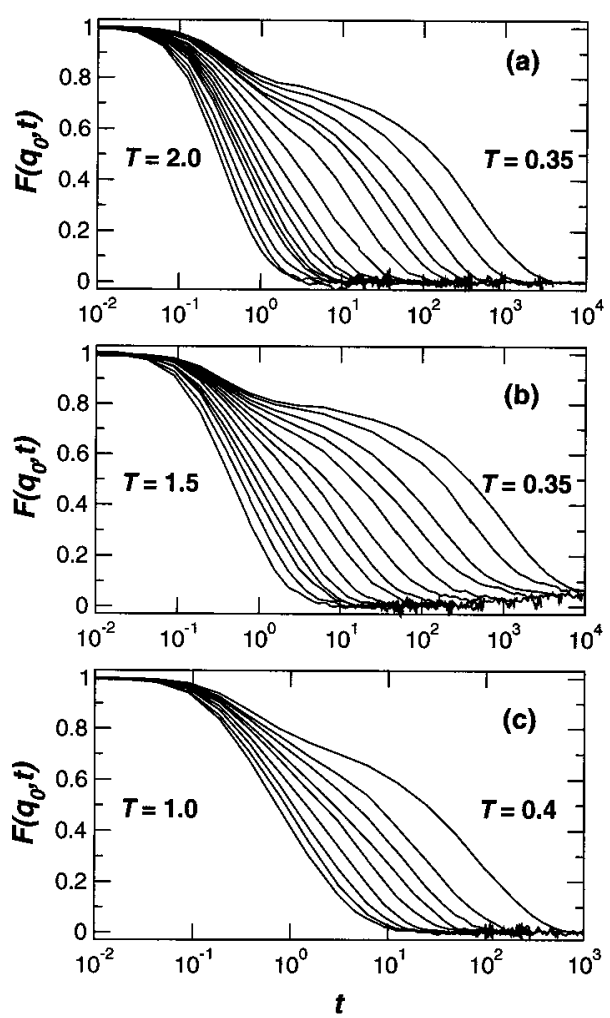

Figure 6. Intermediate scattering function $F\left(q_{0}, t\right)$ for all state points studied with $\mathrm{N}_{\mathrm{p}}=400$. (a) Pure system at $\mathrm{T}=2.0,1.5$, $1.25,1.1,1.0,0.9,0.8,0.7,0.6,0.5,0.45,0.42,0.4,0.37$, and 0.35 . (b) Attractive nanoparticle system at $\mathrm{T}=1.5,1.2,1.0$, $0.9,0.8,0.7,0.6,0.55,0.5,0.45,0.42,0.4,0.37$, and 0.35 . (c) Nonattractive nanoparticle system at $\mathrm{T}=1.0,0.9,0.8,0.7$, $0.6,0.550 .5,0.45$, and 0.4 .

ture both from physical intuition and on theoretical grounds. ${ }^{41,42}$

\section{Effect of Nanoparticle on Melt Dynamics}

4.1. Effect of Polymer-Particle Interactions. To quantify the effect of the nanoparticle on $\mathrm{T}_{\mathrm{g}}$ and on dynamic properties, we first calculate the relaxation time $\tau$ of the radially-averaged intermediate scattering function

$$
F(q, t) \equiv \frac{1}{N S(q)}\left(\sum_{j, k=1}^{N} e^{-i q \cdot\left[r_{k}(t)-r_{j}(0)\right]}\right)
$$

We normalize by the structure factor $S(q)$ such that $F(q, 0)=1$. The characteristic time for the decay of $F(q, t)$ diverges as $\mathrm{q} \rightarrow 0$ and has a local maximum at $\mathrm{q}_{0} \approx$ 7.08 , the location of the first maximum in $\mathrm{S}(\mathrm{q})$. We show $F\left(q_{0}, t\right)$ for several $T$ for each system studied in Figure 6. At sufficiently low $T$, each system shows a two-step relaxation of $F(q, t)$. Notice that for the filled attractive system, where the relaxation at a given $\mathrm{T}$ is slowest, $F\left(q_{0}, t\right)$ does not fully decay to zero at the lowest temperatures. This indicates that there is a very slow component of the relaxation; indeed, as we will show later, this appears to be attributable to slowed dynamics near the surface of the nanoparticle.

To facilitate comparison of the relaxation between systems, we next focus on the relaxation time $\tau$, which we define by $F(q, \tau) \equiv 0.2$. The exact choice for the definition of $\tau$ does not qualitatively affect our results. Relative to the pure system, we find that $\tau$ is larger at 


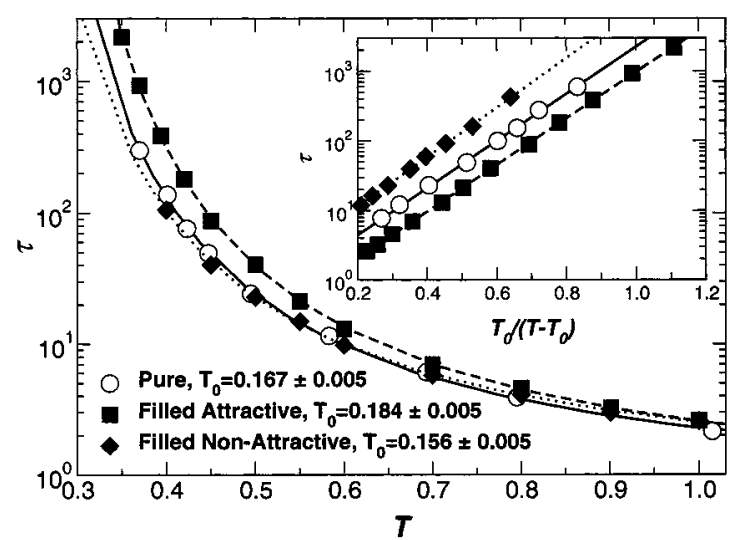

Figure 7. Temperature dependence of the relaxation time of the intermediate scattering function. The lines are a fit to the VFT form of eq 6 . The inset shows the same data plotted against reduced temperature $T_{0} /\left(T-T_{0}\right)$ to show the quality of the VFT fit. For clarity in the inset, $\tau$ of the pure system is multiplied by 2 , and $\tau$ of the filled nonattractive system is multiplied by 4 .

each $\mathrm{T}$ for the attractive system, and this difference increases with decreasing $T$ (Figure 7). Hence, one expects that the attractive system has a higher $\mathrm{T}_{\mathrm{g}}$. Conversely, $\tau$ in the nonattractive system is nearly indistinguishable from that in the pure system at high $\mathrm{T} ; \tau$ in the nonattractive system is slightly smaller at low $\mathrm{T}$ than in the pure system, and this difference increases with decreasing $T$. Thus, we expect the nonattractive system to have a lower $\mathrm{T}_{\mathrm{g}}$ than the pure system.

As a quantitative check on the expected $T_{g}$ shift, we fit $\tau$ to the Vogel-Fulcher-Tammann (VFT) form

$$
\tau \sim \mathrm{e}^{\mathrm{A} /\left(\mathrm{T}-\mathrm{T}_{0}\right)}
$$

where $T_{0}$ is typically quite cl ose to the experimental $T_{g}$ value, ${ }^{43}$ provided the data used to fit eq 6 are at sufficiently low $\mathrm{T}$; hence, changes in $\mathrm{T}_{\mathrm{g}}$ are reflected in $\mathrm{T}_{0}$. Consistent with the changes in $\tau$ relative to the pure melt, we find that $T_{0}$ increases in the system with attractive interactions but decreases in the system with only an excluded-volume interaction. Thus, the effect of the steric hindrance introduced by the nanoparticle decreases $\tau(T)$ and $T_{0}$, although monomers have a reduced number of directions in which to move, and hence degrees of freedom that aid in the loss of correlations. The fact that $\mathrm{T}_{0}$ shifts in opposite directions for attractive vs purely excluded-volume interactions demonstrates the importance of the surface interactions, which we will probe further in the following.

4.2. Effect of Concentration. Experiments on filled polymer melts have shown that $\mathrm{T}_{\mathrm{g}}$ shifts become more pronounced as the filler concentration increases.1,9-11 However, simulating many nanoparticles in the melt requires system sizes substantially larger than we consider. However, we can trivially see some of the effects of concentration in the present simulations by varying the number of polymers surrounding the nanoparticle. This will show us the effect of changing the surface-to-volume ratio but does not provide insight into the possible changes due to interactions of polymers with multiple nanoparticles, such as chain bridging between nanoparticles or confinement.

Figure 8 shows estimated $T_{0}$ values for each system size studied. As the mass fraction of nanoparticle (and thus surface-to-volume ratio) increases, the shift of $\mathrm{T}_{0}$

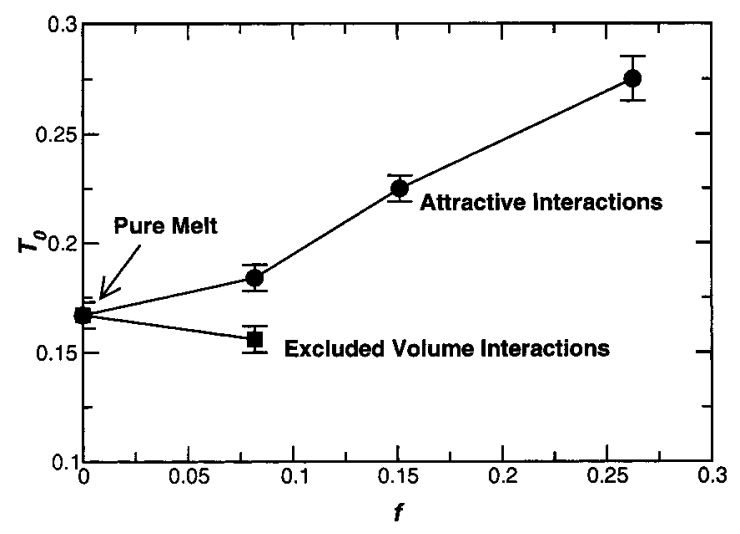

Figure 8. Effect of changing the surface-to-volume ratio on $\mathrm{T}_{\mathrm{g}}$. Here, $\mathrm{f}$ is the mass fraction of nanoparticle, so increasing $f$ corresponds to an increasing surface-to-volume ratio. The error bars estimate the range of $\mathrm{T}_{0}$ values that can be used in eq 6 to fit to the data.

becomes more pronounced. This is consistent with the hypothesis that the $T_{g}$ shifts result from changes in the dynamics in the vicinity of the surface. The next section focuses on characterizing these surface dynamics.

4.3. Dynamics Approaching Nanoparticle Surface. To elucidate how the polymer dynamics are influenced by the interactions with the surface, we probe the melt dynamics as a function of distance from the nanoparticle surface. This can be done in the context of $F(q, t)$ but is more clearly understood by limiting ourselves to the self (or incoherent) part $F_{\text {self }}(q, t)$ of $F(q, t)$. To examine the rel axation properties of monomers near the surface, and as a function of distance from the surface, we calculate $F_{\text {self }}(q, t)$ on the basis of the position of a monomer at $\mathrm{t}=0$. To further simplify this without loss of generality, we utilize the fact that monomers form well-defined layers surrounding the nanoparticle, as shown in Figure 3; hence, we split $F_{\text {self }}(q, t)$ into the contribution from monomers located in each layer at $t$ $=0$. In this way, we decompose

$$
\mathrm{F}_{\text {self }}(\mathrm{q}, \mathrm{t})=\frac{1}{\mathrm{~N}} \sum_{\text {layers }} \mathrm{N}_{\text {layer }} \mathrm{F}_{\text {self }}^{\text {layer }}(\mathrm{q}, \mathrm{t})
$$

where $\mathrm{N}_{\text {layer }}$ is the number of monomers in a given layer. We show $F_{\text {self }}^{\text {layer }}\left(q_{0}, t\right)$ as well as $F_{\text {self }}\left(q_{0}, t\right)$ for $T=0.4$ in Figure 9.44 In the attractive system, the relaxation of the layers closest to the nanoparticle are slowest, consistent with the system dynamics being slowed by the attraction to the nanoparticle and the increase in $\mathrm{T}_{\mathrm{g}}$. Conversely, for the nonattractive system, we find that the relaxation of surface layer monomers is significantly enhanced compared to the bulk, consistent with the decrease of $T_{g}$. The altered dynamics persist for a distance slightly less than $2 R_{g}$ from the surface. We also show in Figure 10 that the relaxation of $F_{\text {self }}^{\text {layer }}\left(q_{0}, t\right)$ for monomers farthest from the nanoparticle surface is nearly coincident with $\mathrm{F}_{\text {self }}\left(\mathrm{q}_{0}, \mathrm{t}\right)$ of the pure system, for either choice of monomer-particle interactions, indicating that at large distances bulklike behavior is recovered. Our results demonstrate that interactions play a key role in controlling $\mathrm{T}_{\mathrm{g}}$ and the local dynamics of polymer/nanoparticle mixtures.

We further probe the effect of attractive interactions by performing a simulation with a stronger monomerparticleattraction $\left(\epsilon_{\mathrm{mp}}=2\right)$ and another simulation with a weaker attraction $\left(\epsilon_{\mathrm{mp}}=0.5\right)$. Figure 11 shows the 

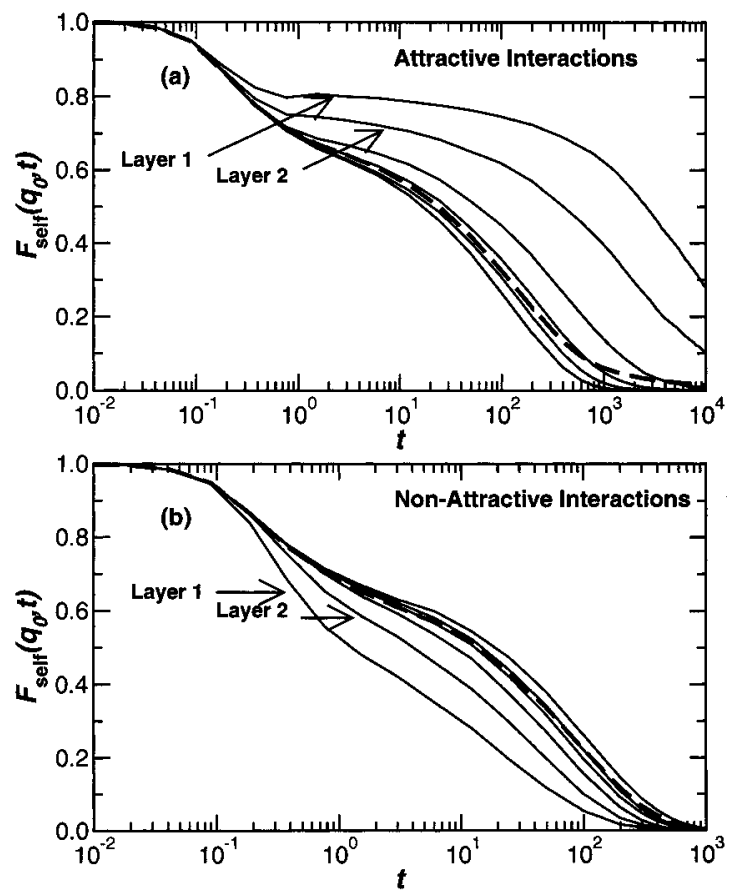

Figure 9. $\mathrm{F}_{\mathrm{self}}\left(\mathrm{q}_{0}, \mathrm{t}\right)$ for the average of all monomers (dotted line) and decomposed into layers (defined by the distance from the nanoparticle surface) for (a) attractive interactions and (b) nonattractive interactions at $T=0.4$. We define the layers using the monomer density profile $\rho\left(d /\left\langle R_{a}\right\rangle\right)$, as shown in Figure 3. We use the minima in $\rho(r)$ to define the boundary between layers. In (a), we see that the relaxation near the nanoparticle surface is sl owed by roughly 2 orders of magnitude. In contrast, (b) shows the relaxation of $F_{\text {self }}\left(q_{0}, t\right)$ is enhanced by roughly 1 order of magnitude near the surface. The relaxation time of the outermost layer in both cases nearly coincides with the relaxation time of the pure system.

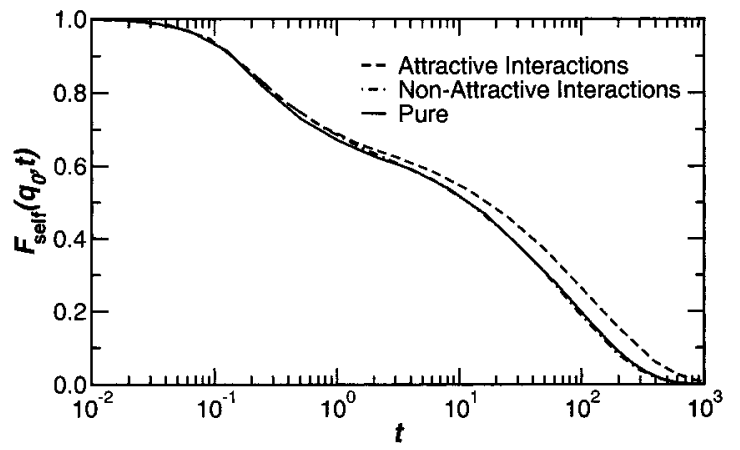

Figure 10. $F_{\text {self }}\left(q_{0}, t\right)$ for the outermost monomers for both choices of partide-polymer interactions compared with $F_{\text {sef }}\left(q_{0}, t\right)$ of the pure system.

results for $F_{\text {self }}^{\text {layer }}\left(q_{0}, t\right)$ for each of these systems at $T=$ 0.4 . The system with strong interactions shows significantly slower relaxation of $F_{\text {self }}^{\text {layer }}\left(q_{0}, t\right)$ near the nanoparticle surface, and these monomers do not fully relax on a time scale that is reasonably accessible to our simulations; as a result, we expect that the increase in $\mathrm{T}_{\mathrm{g}}$ is greater than that observed for weaker attractions. On the other hand, when the attraction is made much weaker, the monomers near the surface show a more rapid vibrational dephasing than the average and slightly slower dynamics in the primary $(\alpha)$ relaxation of $F_{\text {self }}^{\text {layer }}\left(q_{0}, t\right)$. Hence, it might be possible that $T_{g}$ could shift to lower $\mathrm{T}$, even when there are attractive monomer-particle interactions, provided they are quite weak.
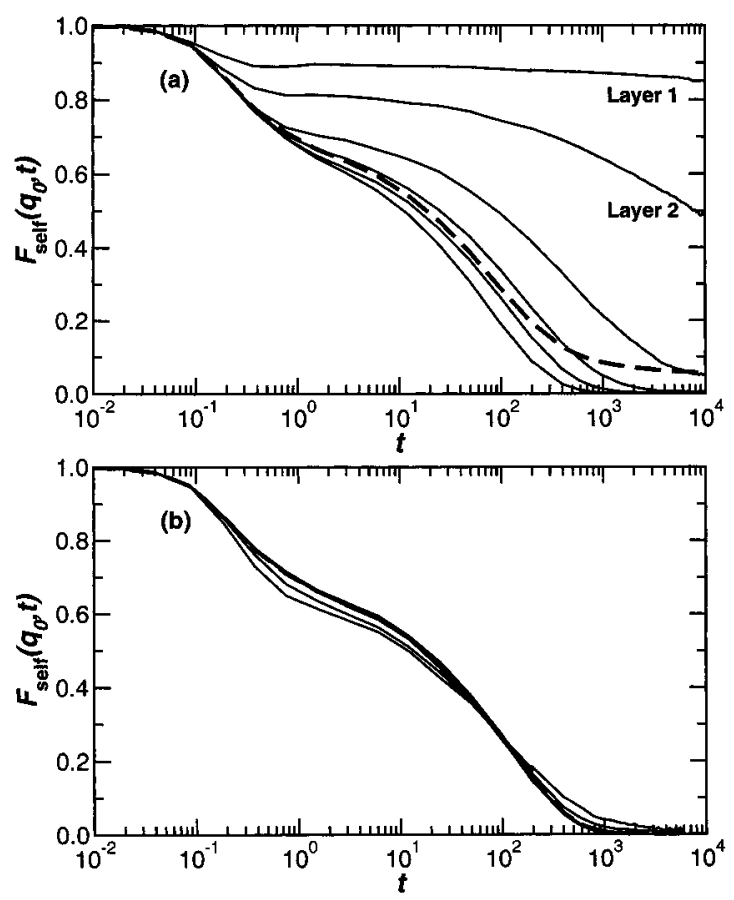

Figure 11. (a) $F_{\text {self }}\left(q_{0}, t\right)$ for the average of all monomers (dotted line) and decomposed into layers for a stronger polymer-particle attraction, $\epsilon_{\mathrm{mp}}=2$. The relaxation near the nanoparticle surface is significantly slower than with weaker polymer-particle attraction. (b) $F_{\text {self }}\left(q_{0}, t\right)$ decomposed into layers for very weak polymer-particle attraction, showing that the surface monomer dynamics, while very similar to the bulk, are slightly altered.

Since we know that the monomers order in layers around the nanoparticle and the chain structure is nonisotropic near the nanoparticle surface, we wish to know whether there is an accompanying anisotropy in the dynamics of the monomers. More specifically, is there an increased tendency for monomers to remain within a specific layer, with relatively infrequent layer changes? This can be easily addressed by decomposing

$$
F_{\text {self }}(q, t)=F_{\text {self }}^{\perp}(q, t) F_{\text {self }}^{\|}(q, t)
$$

where $\mathrm{F}_{\text {self }}^{\perp}(\mathrm{q}, \mathrm{t})$ is the radial component relative to the nanoparticle center, roughly perpendicular to the nanoparticle surface, and $F_{\text {self }}^{\|}(q, t)$ combines the angular components, roughly parallel to the nanoparticle surface. The component $F_{\text {self }}^{\perp}(q, t)$ is obtained by substituting $\mathbf{r}_{\mathrm{k}}(\mathrm{t})-\mathbf{r}_{\mathrm{j}}(0)$ of eq 5 with the radial component, analogous to the calculation of $R_{g}^{\perp}$ in eq 4 . In the normalization, $\mathrm{S}(\mathrm{q})$ must also be broken into components in the same fashion.

If the relaxation is isotropic, then we expect $F_{\text {self }}(q, t)$ $=\left(F_{\text {self }}^{\perp}(q, t)\right)^{3}$. Alternatively, if there is a decreased tendency for monomer exchange between layers, $\left(F_{\text {self }}^{\perp}(q, t)\right)^{3}>F_{\text {self }}(q, t)$; i.e., the correlations involving motions between layers will not have relaxed as much as the correlations for motion within a layer. Figure 12 shows that at $\mathrm{T}=0.4 \mathrm{~F}_{\text {self }}\left(\mathrm{q}_{0}, \mathrm{t}\right) \approx\left(\mathrm{F}_{\text {self }}^{\perp}\left(\mathrm{q}_{0}, \mathrm{t}\right)\right)^{3}$, but $\left(F_{\text {self }}^{\perp}(q, t)\right)^{3}$ is slightly larger for sufficiently large $t$. Hence, there is a slight increased relative tendency for monomer motion to occur within the layer rather than between layers.

In parallel with Figure 8, we also show how $F\left(q_{0}, t\right)$ varies as a function of the system size for $T=0.5$ (Figure 13). As we decrease the system size, the ratio 


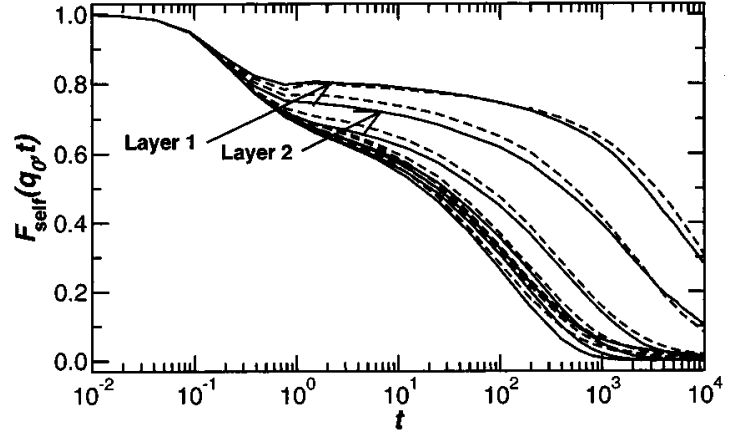

Figure 12. $F_{\text {self }}\left(q_{0}, t\right)$ by layer (solid line), and decomposed into the perpendicular component $\left.F_{\text {self }}^{\perp}(q, t)\right)^{3}$ (dotted line), showing that the relaxation is nearly isotropic, in marked contrast with the anisotropic structure of the chains shown in Figure 4.

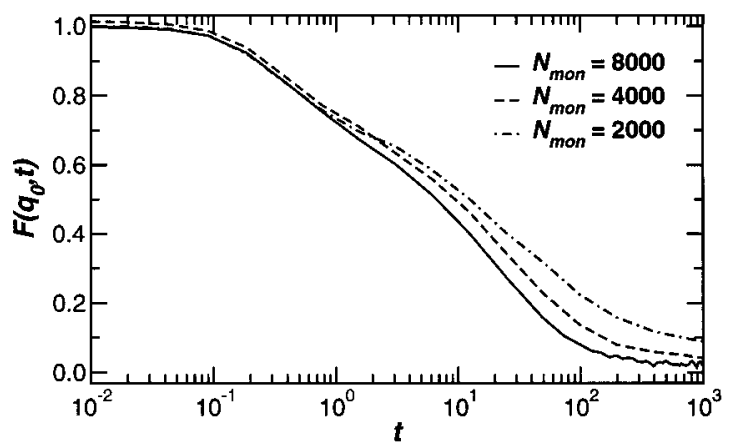

Figure 13. $F\left(q_{0}, t\right)$ at $T=0.5$ for each system size studied.
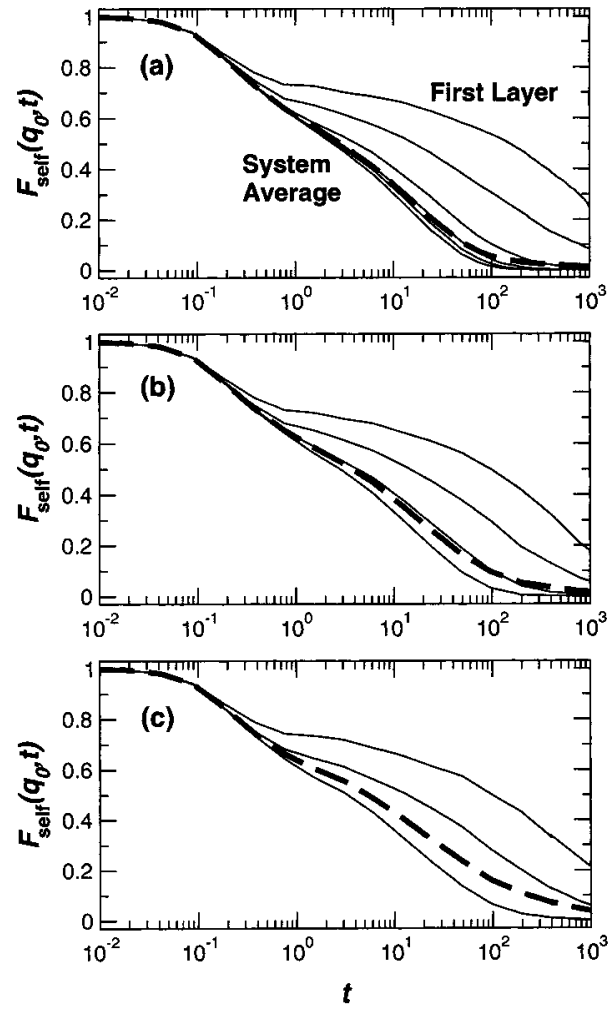

Figure 14. $F_{\text {self }}\left(q_{0}, t\right)$ by layer for system sizes $(a) N=8000$, (b) 4000 , and (c) 2000. In each figure, the system average is indicated by a dashed line, and the relaxation of the layer nearest the surface is the uppermost curve.

of surface to volume increases, and so $F\left(q_{0}, t\right)$ decays more slowly. However, if we consider the relaxation of each layer (Figure 14), we see that each layer relaxes more or less identically as we decrease the system size,

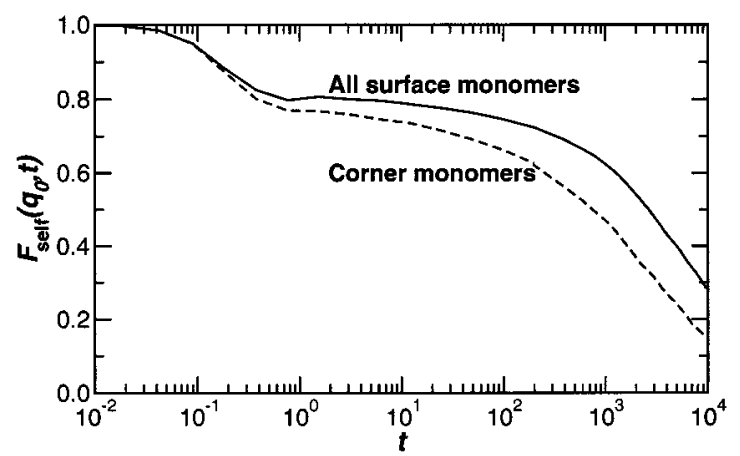

Figure 15. $\mathrm{F}_{\text {self }}\left(\mathrm{q}_{0}, \mathrm{t}\right)$ for all surface monomers (layer 1 of Figure 9a) and for those monomers at the corners at $\mathrm{T}=0.4$.

except that the number of layers is reduced.

4.4. Effect of Geometry: Dynamics near a Corner. Our simulations focus on the effects of a single nanoscopic particle surrounded by an unentangled melt of polymer chains. A real polymer-particle system, such as a filled polymer, contains many particles, and so there may be effects due to the interactions and geometry of multiple particles that we cannot capture in the present study. We expect the effect of interactions near the particle surface to be largely the same when many nanoparticles are present in the melt, but we expect additional effects on dynamic properties due to the more complex geometrical constraints. However, the dispersion of particles in the melt will be sensitive to the interactions, which will in turn affect the overall melt properties. This is an important area of future study. In the present system, we can explore the limited effect of the geometry by studying, e.g., the dynamics of monomers near the nanoparticle corners.

We calculate $F_{\text {self }}\left(q_{0}, t\right)$ for those monomers that are initially within one monomer spacing of a corner of the nanoparticle. In Figure 15, we see that the relaxation of the corner monomers is qualitatively very similar to other surface monomers but somewhat faster. This can be expected since, at the corners, there are more free directions for a monomer to move than at a face of the nanoparticle. This supports the expectation that interactions will have a similar effect for more geometrically complicated orientations.

\section{Relationship to Ultrathin Films}

As we hinted at earlier, our results seem applicable not only to nanoparticle/polymer systems but also to nearly any polymer system where surface interactions are important. One pertinent example is ultrathin polymer films (thickness $\leqslant 100 \mathrm{~nm}$ ), where there is longstanding debate on the role of interactions vs confinement on $\mathrm{T}_{\mathrm{g}}$ shifts, ${ }^{14,22,23}$ local melt dynamics, ${ }^{14,24-28}$ and melt structure. ${ }^{14-20}$ Our simulations allow us to address the effects of interactions with a surface, without the additional complication of confinement effects present in thin films. It is largely agreed that ultrathin films on strongly attractive substrates increase $\mathrm{T}_{\mathrm{g}}$, while weak substrate interactions (or no substrate, as in freely standing films) lead to a downward shift of $\mathrm{T}_{\mathrm{g}}$; this is consistent with our results. This consistency is reasonable for particles with facets that are relatively smooth and large compared to $\mathrm{R}_{\mathrm{g}}$; for nanoscopic particles, such as we study, it is interesting that a correspondence occurs even for $\mathrm{R}_{\mathrm{g}}$ close to the nanoparticle size.

Not surprisingly, the magnitude of the shifts we observe depends on the relative quantities of polymer 
and nanoparticle; a greater nanoparticle concentration would have a more dramatic effect (as observed experimentally in refs 9-11). Insofar as the magnitude of effects depends only on the ratio of the surface to bulk monomers, the thickness of the film is analogous to the inverse of the concentration of the nanoparticles. This is consistent with the experimental observation that $T_{g}$ shifts are more pronounced as film thickness decreases. Recently, there have been several experiments on segmental motion in both freely standing and supported ultrathin films. 14,24,25 The observed speedup of segmental dynamics is consistent with a decreased $\mathrm{T}_{\mathrm{g}}$ found in calorimetric measurements ${ }^{14,22,23}$ At this time, it is not clear whether a model with layers of different mobility is applicable to understand $\mathrm{T}_{\mathrm{g}}$ shifts of thin films; ${ }^{14}$ however, the parallel behavior we observe between the thin films and our simulations of a polymer-particle system supports this viewpoint. Finally, the elongation and flattening of polymers we observe near the nanoparticle have been observed in thin-film simulations ${ }^{17-20}$ as well as recent experiments; ${ }^{15,16}$ the range of the effect found in ref 15 is quantitatively consistent with our results, which show the effect only for a range of roughly $R_{g}$, while the results of ref 16 observed flattening for film thicknesses $\leqslant 6 R_{\mathrm{g}}$. We also found, as in ref 15 , that the chains retain a Gaussian structure near the surface, since $\mathrm{R}_{\mathrm{e}}{ }^{2} \approx 6 \mathrm{R}_{\mathrm{g}}{ }^{2}$. Thus, our findings demonstrate that confinement is not a necessary ingredient for the observed changes in the dynamics and structure of polymers near surfaces. While our results provide strong support for interpreting the results for particle/polymer mixtures, filled melts, and ultrathin films in the same general framework, it is obvious that much care must be used when analyzing specific systems.

\section{Effect of a Free Surface}

From an experimental standpoint, it is difficult to explicitly study the properties of surface polymers in a filled melt; ultrathin films avoid this complication, since nearly all monomers are near a surface. For filled systems, pure "unbound" polymers are solvent extracted, using methods such as Soxhlet extraction, which leave only a thin layer of "bound" polymer which strongly adheres to the particle surface. ${ }^{1,45,46}$ The removal of the bulk material facilitates studying the properties of polymers near the nanoparticle surface but also leaves a free surface. Similarly, for supported or freely standing films, at least one surface of the film is free. Hence, it is not immediately clear how our results can relate to these more complicated experimental situations.

While we do not attempt to simulate the chemical process of chain extraction that yields bound polymer, we can gain some insight into what differences there may be between the results we have presented and what is experimentally observed when there is a free surface. In the initial study, we took great care to set up the simulation so that the melt density far from the surface has always the same value. This prevents any significant changes due to differences in the overall density. However, in an experiment, the free boundary and constant pressure will result in a T-dependent density far from the particle. We can introduce similar conditions by simply removing the periodic boundary conditions of our original simulations, ${ }^{47}$ such that the outermost chains are in contact with a free surface (for the attractive nanoparticle $\left.{ }^{48}\right)$. Whether or not the chains

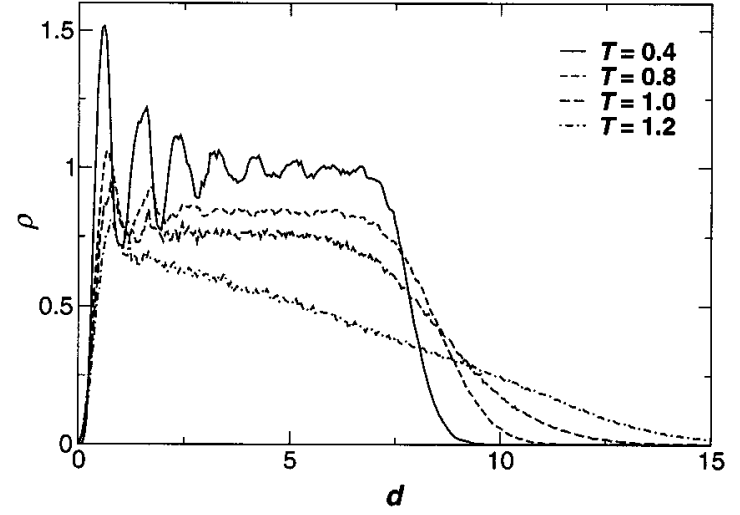

Figure 16. Density profile of the bound monomers for various $\mathrm{T}$. For clarity, only a selection of $\mathrm{T}$ is shown.

will leave the nanoparticle surface will depend on T. F or $\mathrm{T} s \epsilon$, no polymers will separate from the nanoparticle, since both the monomer-monomer and monomerparticle attraction exceeds $T$ (neglecting the entropic contribution to the free energy). For $\epsilon \lesssim T \leqslant \epsilon_{\mathrm{mp}}$, some chains do escape, but others remain near the nanoparticle surface due to the stronger monomer-particle attraction. For $T \gtrsim \epsilon_{\mathrm{mp}}$, all chains evaporate from the nanoparticle surface. This is similar to the experimental observation that the amount of bound rubber depends on the temperature at which the extraction is performed. ${ }^{45}$ At large enough $\mathrm{T}$, all the rubber is extracted as the monomer-particle interaction strength is exceeded. It should be noted that higher molecular weights, where entanglement becomes significant, will only evaporate at higher $\mathrm{T}$.

6.1. Bound Polymer Structure. We calculate the density profile for $\mathrm{T}=0.4,0.5,0.6,0.8,1.0,1.2$, and 1.5. In the case of the bulk system, the density profile had very little $T$ dependence, while for the bound polymers we see that $T$ plays a very important role (F igure 16), as expected due to the competition between attraction and temperature. As T increases, the attraction to the nanoparticle surface plays an increasingly less important role, and the thickness of the polymer layer surrounding the particle increases. For T > 1.0, we see the kinetics dominate, and some chains leave the nanoparticle surface, resulting in a less well-defined interface between the bound and unbound chains. At $T$ $=1.5$, no chains remain at the nanoparticle surface, and the polymer forms a diffuse gas of chains.

We can estimate the thickness of the bound layer as a function of $\mathrm{T}$ by calculating the distance at which $\rho(\mathrm{d})$ vanishes. However, because of fluctuations of the outermost chains, this value is not very reliable. Hence, we estimate the thickness by the distance at which $\rho(d)$ $=0.01$, a small but reproducible value of density. As expected from Figure 16, Figure 17 shows that the thickness increases with T. For freely standing films, it has been predicted that the film thickness should increase linearly with $T$ when the film is not glassy; 49 in the range $0.4<\mathrm{T}<1.5$, where the polymers are not glassy, we find that the thickness is not a linear function of $\mathrm{T}$ and can instead be well-approximated by a quadratic expression. The significance of a quadratic form for the thickness and the constants required in the quadratic is unclear. It is possible that we do not observe linear behavior because the dynamics in the $T$ range we study are significantly anharmonic and the molecular weight is small. At lower $\mathrm{T}$ (closer to but above $\mathrm{T}_{\mathrm{g}}$ ) 


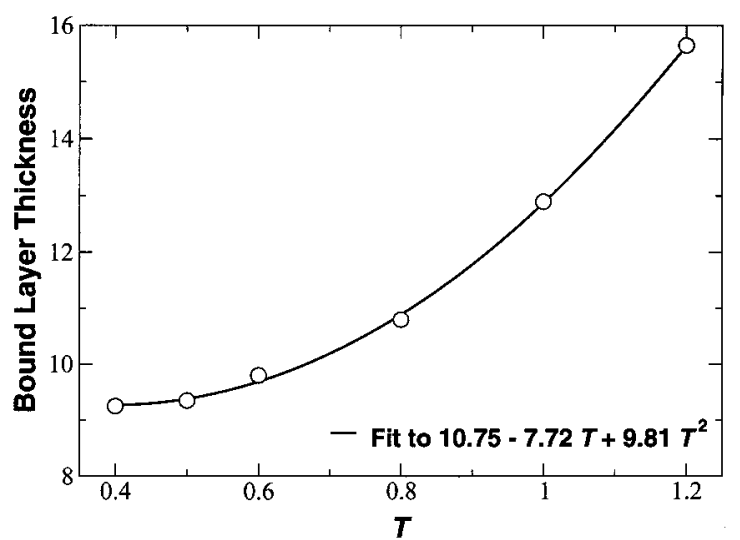

Figure 17. Thickness of the bound layer as a function of $T$.

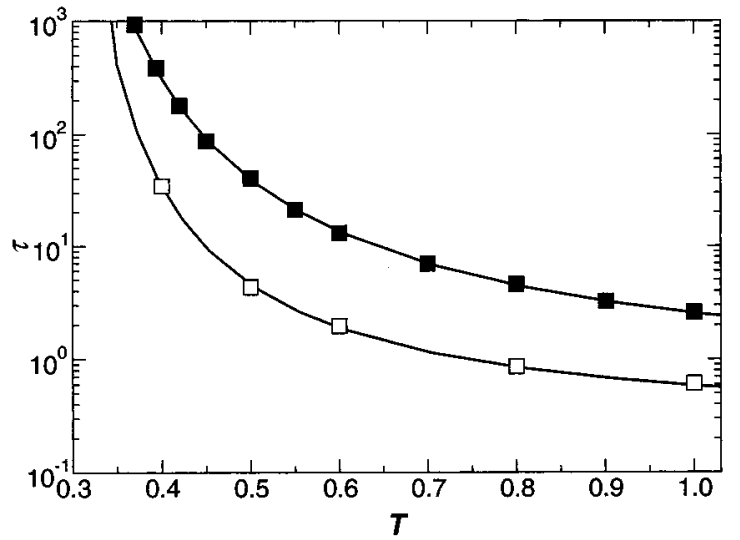

Figure 18. Temperature dependence of the relaxation time $\tau$ of $F\left(q_{0}, t\right)$ for the bound monomers (open $\square$ ) and for the bulk system (filled $\square$ ). In both cases, the nanopartide has attractive interactions.

it is possible that linear behavior may be observed, but such simulations are beyond the scope of the present study.

We checked the effect of increasing the strength of the monomer-particle attraction; increasing the monomer-particle attraction alters the density profile at small distances but has little effect on the density at larger distances. Additionally, the temperature at which all chains will unbind from the nanoparticle surface must also be higher, due to the increased attraction. Hence, for our system, the range, rather than the strength, of the attraction appears more important for the thickness of the polymer layer surrounding the nanoparticle. However, we point out that our chains are below the entanglement threshold; entanglement of outer layer chains with more strongly bound surface chains may show qualitatively different results when the interaction strength is changed.

6.2. Bound Polymer Dynamics. We first explore the change in dynamics of the bound polymers by comparing the relaxation time $\tau$ of $\mathrm{F}\left(\mathrm{q}_{0}, \mathrm{t}\right)$ with that of the bulk system (Figure 18). We find that at each $T$ the relaxation time of the bound system is significantly smaller than that of the bulk system; however, at low $\mathrm{T}, \tau$ appears to increase far more quickly (in other words, the system appears more fragile ${ }^{50}$ ). Correspondingly, using a VFT fit (eq 6), we estimate $T_{0}=0.25 \pm$ 0.02 for the bound system, larger than $T_{0}=0.184 \pm$ 0.006 that we estimated for the bulk system.

The fact that $\tau$ is significantly smaller at each $\mathrm{T}$ for the bound polymers can be rationalized by again exam-

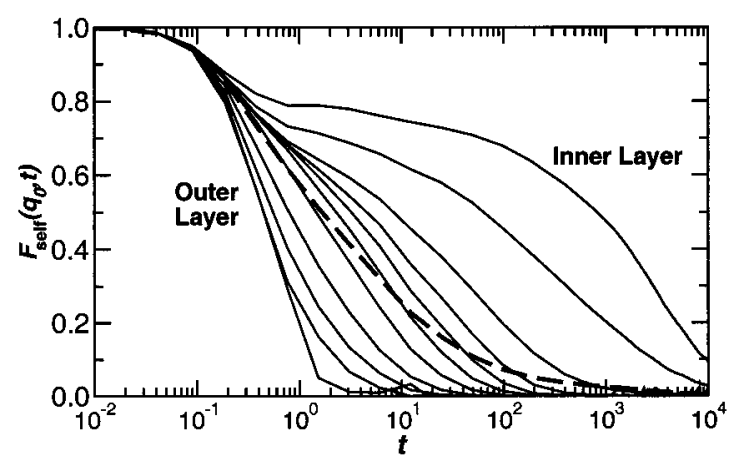

Figure 19. $\mathrm{F}_{\text {self }}\left(\mathrm{q}_{0}, \mathrm{t}\right)$ for the bound monomers by layer at $\mathrm{T}=$ 0.4 . The system average is indicated by the dashed curve.

ining $F_{\text {self }}\left(q_{0}, t\right)$. Figure 19 shows that the layers closest to the nanoparticle surface are dramatically slowed, as we previously observed in the bulk system. However, as we approach the free surface of the bound polymers, the relaxation of $F_{\text {self }}\left(q_{0}, t\right)$ is dramatically enhanced, such that the relaxation time of monomers in the outer layer is roughly 4 orders of magnitude faster than that of the innermost monomers. ${ }^{44}$ As a result (since there are many more monomers in the outermost layer than the innermost layer), the overall relaxation time of the system is reduced. The fact that the outermost monomers relax more quickly is not surprising since the monomers are unbounded on one side and thus can explore space with far fewer constraints on their motion. Indeed, enhanced chain mobility near free surfaces has been observed previously. ${ }^{14}$

\section{Discussion}

By varying the polymer/particle interactions and the surface-to-vol ume ratio, we are able to rationalize the observed changes in the dynamic properties due to surface interactions. Moreover, our results suggest that tentative predictions can be made for the structure and dynamics of systems where a relatively simple surface is present. The changes in surface dynamics give rise to the expectation that attractive interactions will increase $T_{g}$ while very weak interactions can suppress $\mathrm{T}_{\mathrm{g}}$. Such predictions are consistent with experimental observations in thin films. However, by also considering the free surface problem, we have seen that these predictions can be difficult to apply.

For systems with both a well-defined and a free surface, such as bound polymers and supported films, the interactions at each surface differ. The resulting large variation of the relaxation time between the two surfaces makes it difficult to predict how $\mathrm{T}_{\mathrm{g}}$ will be affected for a given polymer-particle interaction strength and range. In the case of $F$ igure 19, if the bound polymer region is thin (very few layers), $T_{g}$ will likely increase with attractive monomer-particle attractions. For a larger thickness of bound polymers (similar to the number of layers we observe), where outer layers of monomer relax far more quickly that inner monomers, $T_{g}$ will likely decrease. Furthermore, for a case with very thick bound polymer, one can imagine that such a disparity in time scales between the inner and outer layers could lead to the presence of two (or more) detectable glass transitions. Hence, while our results provide a clear indication of the effects of a single nanoparticle, the overall result on bulk properties will depend significantly on the specific system properties. 
When interpreting changes in the dynamic properties in a system with confinement of interfering surfaces, there are at least two obvious causes to be considered: (i) changes in local dynamics due to surface interactions and (ii) changes in gl obal dynamics due to "interference" with "important" length scales. Generally, we have interpreted the dynamical changes in terms of a local layer based model, where the dynamics near a surface are altered by the surface interactions and bulk behavior is recovered sufficiently far from the surface. This interpretation appears robust for our results, since far from the nanoparticle surface the monomer dynamics are nearly identical to that of the pure system (as measured by the self-intermediate scattering function in Figure 10). However, recent studies on the spatially heterogeneous dynamics of polymer melts indicate the existence of a growing length scale on cooling, connected to the increased range of collective motion in the system. ${ }^{51,52}$ In the T range we consider, the length scale of this motion is still smaller than the separation of the periodic images of the nanoparticle, ${ }^{52}$ and so focusing only on local changes near surfaces is appropriate. However, at lower $\mathrm{T}$, when the length scale of correlated motion is larger, one must be careful to separate those effects that are local changes due to surface interactions from those that are global changes caused by interfering with naturally occurring length scales in the system. Hence, using only a layer-based model to explain changes in the experimentally measured $\mathrm{T}_{\mathrm{g}}$ may not be sufficient to understand the physics of confined systems near $\mathrm{T}_{\mathrm{g}}$.

The parameters of our simulations were chosen such that the density far from the nanoparticle surface is the same as that of the pure melt we compare with. This was done to limit the source of structural and dynamic changes to the geometry and interactions of the nanoparticle. Experimentally, it is far more common that the pressure remains constant, and hence density varies. Relatively small changes in density can result in significant changes in the bulk dynamics and glass transition temperature. Hence, it is natural to ask whether the results here have rel evance to a system at constant pressure. Clearly, the qualitative changes in surface dynamics, dominated by interactions, will be similar whether or not density changes. For bulk properties, the situation is not so obvious. At constant pressure, the addition of an attractive nanoparticle will result in an increased density. For most systems, $T_{g}$ increases with increasing density, and so the increase of $T_{g}$ we observe would be amplified. Thus, our results should also be useful for interpreting changes at constant pressure, but a direct comparison would be useful to identify the quantitative differences between the systems.

Acknowledgment. We thank E. Amis, J . Douglas, Y. Gebremichael, C. Han, A. Karim, N. Lačević, A. Nakatani, and W. Wu for useful discussions.

\section{References and Notes}

(1) Wypych, G. Handbook of Fillers; ChemTec Publishing: Toronto, 1999.

(2) Donnet, J .-B., Bansal, R. C., Wang, M.-J ., Eds.; Carbon Black: Science and Technology; Marcel Dekker: New York, 1993.

(3) Roco, M. C., Williams, S., Alivisatos, P., Eds.; Nanotechnol ogy Research Directions: IWGN Workshop Report Vision for Nanotechnol ogy in the Next Decade; Kluwer Academic Publishers: Dordrecht, 2000.
(4) Carrot, G.; Valmalette, J . C.; Plummer, C. J . G.; Scholz, S. M.; Dutta, J .; Hofmann, H.; Hilborn, J . G. Colloid Polym. Sci. 1998, 276, 853-859. Cole, D. H.; Shull, K. R.; Baldo, P.; Rehn L. Macromolecules 1999, 32, 771-779. Caruso, F.; Mohwald, H. Langmuir 1999, 15, 8276-8281. Sarathy, K. V.; Narayan, K. S.; Kim, J .; White, J . O. Chem. Phys. Lett. 2000, 318, 543548. Gittins, D. I.; Caruso, F. Adv. Mater. 2000, 12, 1947. Watson, K.J .; Zhu, J .; Nguyen, S. T.; Mirkin, C. A. PureAppl. Chem. 2000, 72, 67-72.

(5) Karim, A.; Liu, D. W.; Douglas, J . F.; Nakatani, A. I.; Amis, E. J . Polymer 2000, 41, 8455-8458. Nakatani, A. I.; Chen, W.; Schmidt, R. G.; Gordon, G. V.; Han, C. C. Polymer 2001, 32, 3713-3722. Roberts, C.; Cosgrove, T.; Schmidt, R. G.; Gordon, G. V. Macromolecules 2001, 34, 538-543.

(6) Barnes, K. A.; Karim, A.; Douglas, J . F.; Nakatani, A. I.; Gruell, H.; Amis, E. J . Macromol ecules 2000, 33, 4177-4185. McCarthy, B.; Coleman, J . N.; Curran, S. A.; Dalton, A. B.; Davey, A. P.; Konya, Z.; Fonseca, A.; Nagy, J. B.; Blau, W. J . J. Mater. Sci., Lett. 2000, 19, 2239-2241. Okumura, Y.; I to, K.; Hayakawa, R.; Nishi, T. Langmuir 2000, 16, 1027810280. Lordi, V.; Yao, N.J . Mater. Res. 2000, 15, 2770-2779. Ajayan, R. M.; Schadler, L. S.; Giannaris, C.; Rubio, A. Adv. Mater. 2000, 12, 750. Qian, D.; Dickey, E. C.; Andrews, R. Rantell, T. Appl. Phys. Lett. 2000, 76, 2868-2870.

(7) Feher, F. J .; Soulivong, D.; Eklund, A. G.; Wyndham, K. D. Chem. Commun. 1997, 13, 1185-1186. Schwab, J .J.; Lichtenhan, J. D. Appl. Organomet. Chem. 1998, 12, 707-713.

(8) von Werne, T.; Patten, T. E. J . Am. Chem. Soc. 1999, 121, 7497-7505. Saujanya, C.; Ashamol; Padalkar, S.; Radhaakrishnan, S. Polymer 2001, 42, 2255-2258. Bronstein, L. M Chernyshov, D. M.; Velatsky, P. M.; Wilder, E. A.; Spontak, R. J. Langmuir 2000, 16, 8221-8225. Rong, M. Z.; Zhang, M. Q.; Zheng, Y. X.; Zeng, H. M.; Walter, R.; Friedrich, K. Polymer 2001, 42, 3301-3304. Friedlander, S. K.; Ogawa, K.; Ullman, M.J. Polym. Sci., Polym. Phys. 2000, 38, 2658-2665.

(9) Cousin, P.; Smith, P. J . Polym. Sci., Part B: Polym. Phys. 1994, 32, 459-468.

(10) Tsagaropoulos, G.; Eisenberg, A. Macromolecules 1995, 28, 6067-6077.

(11) Sombatsompop, N.J . Appl. Polym. Sci. 1999, 74, 1129-1139.

(12) Starr, F. W.; Schrøder, T. B.; Glotzer, S. C. Phys. Rev. E 2001 64, 021802.

(13) Vacatello, M. Macromol ecules 2001, 34, 1946-1952.

(14) Forrest, J . A.; J ones, R. A. L. In Polymer Surfaces, Interfaces, and Thin Films; Karim, A., Kumar, S., Eds.; World Scientific: Singapore, 2000; p 251 and references therein.

(15) J ones, R. L.; Kumar, S. K.; Ho, D. L.; Briber, R. M.; Russell, T. P. Nature (London) 2000, 400, 146-149.

(16) Kraus, J .; Müller-Buschbaum, P.; Kuhlmann, T.; Schubert, D. W.; Stamm, M. Europhys. Lett. 2000, 49, 210-216.

(17) Theodorou, D. N. Macromol ecules 1988, 21, 1400-1410.

(18) Kumar, S. K.; Vacatello, M.; Yoon, D. Y. J . Chem. Phys. 1989, 89, 5206-5215

(19) Kumar, S. K.; Vacatello, M.; Yoon, D. Y. Macromolecules 1990, 23, 2189-2197.

(20) Wang, J .-S.; Binder, K. J . Phys. I 1991, 1, 1583-1590.

(21) Mischler, C.; Baschnagel, J .; Binder, K., cond-mat/0012277.

(22) van Zanten, J . H.; Wallace, W. E.; Wu, W. L. Phys. Rev. E 1996, 53, 2053-2056

(23) Wallace, W. E.; van Zanten, J . H.; Wu, W. L. Phys. Rev. E 1995, 52, 3329-3332.

(24) Forrest, J . A.; Dalnoki-Veress, K.; Dutcher, J . R. Phys. Rev. E 1997, 56, 5705-5716.

(25) Forrest, J. A.; Svanberg, C.; Revesz, K.; Rodahl, M.; Torell, L. M.; Kasemo, B. Phys. Rev. E 1998, 58, 1226-1229.

(26) Lin, E. K.; Kolb, R.; Satija, S. K.; Wu, W. L. Macromolecules 1999, 32, 3753-3757.

(27) Varnik, F.; Baschnagel, J .; Binder, K. J . Phys. IV 2000, 10, 239-242.

(28) Anastasiadis, S. H.; Karatasos, K.; Vlachos, G.; Manias, E.; Giannelis, E. P. Phys. Rev. Lett. 2000, 84, 915-918.

(29) Bird, R. B.; Curtiss, C. F.; Armstrong, R. C.; Hassager, O. Dynamics of Polymeric Liquids: Kinetic Theory; J ohn Wiley and Sons: New York, 1987; Vol. 2.

(30) Grest, G. S.; Kremer, K. Phys. Rev. A 1986, 33, 3628-3631.

(31) Rudisill, J. W.; Cummings, P. T. Rheol. Acta 1991, 30, 3343.

(32) Kremer, K.; Grest, G. S. J . Chem. Phys. 1990, 92, 5057-5086.

(33) Bennemann, C.; Paul, W.; Binder, K.; Dünweg, B. Phys. Rev E 1998, 57, 843-851. Bennemann, C.; Paul, W.; Baschnagel J .; Binder, K. J . Phys.: Condens. Matter 1999, 11, 21792192. Bennemann, C.; Baschnagel, J .; Paul, W. Eur. Phys. . . 1999, 10, 323-334. 
(34) Stillinger, F. H.; Weber, T. A. Phys. Rev. A 1982, 25, 978989; Science 1984, 225, 983-989. Stillinger, F. H. Science 1995, 267, 1935-1939.

(35) Sastry, S.; Debenedetti, P. G.; Stillinger, F. H. Nature (London) 1998, 393, 554-557. Sciortino, F.; Kob, W.; Tartaglia, P. Phys. Rev. Lett. 1999, 83, 3214-3217. Buechner, S.; Heuer, A. Phys. Rev. E 1999, 60, 6507-6518. Schrøder, T. B.; Sastry, S.; Dyre, J .; Glotzer, S. C. J . Chem. Phys. 2000, 112, 9834-9840. Scala, A.; Starr, F. W.; La Nave, E.; Sciortino, F.: Stanley, H. E. Nature(London) 2000, 406, 166169. Debenedetti, P. G.; Stillinger, F. H. Nature (London) 2001, 410, 259-267.

(36) Allen, M. P.; Tildesley, D. J . Computer Simulation of Liquids; Oxford University Press: Oxford, 1987.

(37) Doi, M.; Edwards, S. F. The Theory of Polymer Dynamics; Oxford University Press: Oxford, 1988

(38) The length I is chosen to be a multiple of $2^{1 / 6}$ so that nanoparticle force sites are separated by the preferred distance of the Lennard-J ones potential.

(39) For some fillers and nanoparticles, it is expected that interactions with the edges will be stronger than interactions with the face. It is not immediately clear how the qualitative results for such a nanoparticle might differ; this question will be addressed in a separate work

(40) Tuckerman, M.; Berne, B. J .; Martyna, G. J . J . Chem. Phys. 1992, 97, 1990-2001.

(41) Douglas, J F.; Nemirovsky, A. M.; Freed, K. F. Macromolecules 1986, 19, 2041-2054.

(42) Brazhnik, P. K.; Freed, K. F.; Tang, H.J . Chem. Phys. 1994, 101, 9143-9154. Freed, K. F. J. Chem. Phys. 1996, 105, 10572-10582.
(43) Debenedetti, P. G. M etastable Liquids; Princeton University Press: Princeton, 1996

(44) At distances beyond where the layers are clearly observable, we define layers using the typical layer thickness found at smaller distance from the filler surface. This allows us to probe changes in the dynamics at distances beyond where the layers are well defined.

(45) Donnet, J .-B.; Vidal, A. Adv. Polym. Sci. 1986, 76, 103-127.

(46) Rigbi, Z. Adv. Polym. Sci. 1980, 36, 21-68.

(47) In fact, to simplify modification of the simulation code, we do not remove the periodic boundary conditions. Instead, we increase the box size by a factor of 4 (and hence the volume by a factor of 64), so that the chains may separate from the surface and exist as a highly diffuse gas.

(48) Naturally, we can only make the test of chains remaining at the nanoparticle surface for the case of attractive monomerparticle interactions; in the excluded-volume case, all chains will eventually leave the nanoparticle surface, even if the chains themselves remain in the melt state

(49) de Gennes, P. G. Eur. Phys. J . E 2000, 2, 201-203.

(50) Angell, C. A. Science 1995, 267, 1924-1935.

(51) Sillescu, H. J . Non-Cryst. Solids 1999, 243, 81-108. Glotzer, S. C. J. Non-Cryst. Solids 2000, 274, 342-355. Ediger, M. D. Annu. Rev. Phys. Chem. 2000, 51, 99-128.

(52) Bennemann, C.; Donati, C.; Baschnagel, J .; Glotzer, S. C. Nature (London) 1999, 399, 246-249. Doliwa, B.; Heuer, A. Phys. Rev. E 2000, 61, 6898-6908. Gebremichael, Y.; Schrøder, T. B.; Starr, F. W.; Glotzer, S. C. Phys. Rev. E 2001, 65, 051503.

MA010626P 